\title{
Classification of the Gabon SAR mosaic using a wavelet based rule classifier
}

\author{
Marc Simard '(1), Sasan Saatchi(1),Gianfranco DeGrandi('2)
}

\author{
(1)Radar Science and Engineering section, Jet Propulsion Laboratory, Pasadena, California, 91101 \\ simard@bacchus.jpl.nasa.gov \\ (2)Space Applications Institute of the Joint Research Center of CEE, Ispra, Italy.
}

\begin{abstract}
A method is developed for semi-automated classification of SAR images of the tropical forest. Information is extracted using the wavelet transform (WT). The transform allows for extraction of structural information in the image as a function of scale. In order to classify the SAR image, a Desicion Tree Classifler is used. The method of pruning is used to optimize classification rate versus tree size. The results give explicit insight on the type of information useful for a given class.
\end{abstract}

Keywords-SAR, tropical forest, rule, tree, multiscale

\section{INTRODUCTION}

The work presented is part of the Global Rain Forest Mapping project (GRFM). Recent work have shown the potential and use of Radar remote sensing for monitoring of tropical forests [1], [2]. The objective of our team is to produce an automated classification algorithm used for automated mapping and monitoring of tropical forest on a global scale using SAR. We present a method for semiautomated classification of SAR images.

The set of data covers the entire tropical belt with JERS1 SAR data acquired at two dates. It was provided by NASDA (japanese space agency). The resolution of the data was reduced to $100 \mathrm{~m}$ before mosaicking. In this paper, we will focus on a part of Gabon where ground truth is available.

Various methods exist to classify images using training areas. One of the most popular is the "Maximum Likelihood" method (ML). Other methods such as neural networks and decision trees offer advantages related to the underlying assumptions which are not needed in the last two methods as well as the explicit information extraction on the various data contributions. In this paper, the decision tree method was used [3]. The tree uses training samples to grow, based on node impurity measure. A split is chosen $f$ it maximizes the the reduction of impurity $(i(t))$ of the parent node $t$ with respect to the impurity of the splits.

$$
\Delta i(t)=i(t)-p_{l} i\left(t_{l}\right)-p_{r} i\left(t_{r}\right)
$$

$p_{l}$ and $p_{r}$ are the proportion of samples in node $t$ that reach into child nodes $l$ and $r$.

The Gini criterion was chosen, as it was shown to be generally efficient [ 3 ]. It is defined as:

$$
1-\sum_{j} p^{2}(j \mid t)
$$

It uses a rule that assigns a sample randomly selected from the node $t$ to class $j$ with probability $p(j \mid t)$. A split is chosen to maximize $\Delta(t)$. The probability is:

$$
p(J \mid t)=\frac{P_{J} \cdot V_{j}(t) / V_{j}}{\sum P_{J} V_{j}(t) / N_{j}}
$$
1 . Mart: Simard is a Nationil Research Council (U.S.) research
associate. where $P_{J}$ is the prior probability.

However, the tree grows indefinitely until all classes are separated which can lead to a very large tree. Instead, we will grow a reasonable tree ( 7 levels), followed by "pruning" of the tree. The pruning method consists in cutting branches wich are inefficient. The decision is based on the misclassification cost, $R(t)=r(t) p(t)$, with

$$
r(t)=1-\max _{j} p(j \mid t)
$$

where $p(t)$ is the probability that any case fall in node $t$.

Following the misclassification cost, a complexity function is defined [3] that takes into account the number of terminal nodes $n T$. The weakest non-terminal node is the one with minumum

$$
g(t)=\frac{R(t)-R\left(T_{\mathrm{t}}\right)}{n T-1}
$$

The original tree is iteratively cut to produce a sequence of subtrees $T_{k}$ with different complexity. The optimum-size tree is chosen using an independant sample which classes are known. This sample is input into the tree sequence and estimates $R\left(T_{k}\right)$ are produced. The optimum tree is the one with minimum estimate $R\left(T_{k}\right)$.

Multiscale information from the SAR image is extracted using the Wavelet Transform (WT) [4]. The Transform produces a multiscale representation of the original images as well as 3 (orientation) detail images. The detail images, which contain the wavelet coefficients, can be considered as band-pass representation whereas each of the detail image contains information at a finite scale interval. The wavelet coefficients (c) are the components of the original image at a given scale $(a)$ on the wavelet base $(\Psi)$ :

$$
c_{a}=\langle I, \Psi\rangle \text {. }
$$

Thus, structures in the SAR image are separated with respect to their scale. Texture maps are built from the detail images by quadratic addition of the 3 orientation detail images and normalisation by the low resolution local amplitude. The normalisation is computed in order to correct for multiplicative noise contribution [5]. The low-resolution approximation, which is an output of Mallat's algorithm contains and estimate of the local reflectivity which is used to estimate noise level. Normalisation was also shown to enhance edges [6].

\section{APPLiCATION OF THE METHOD}

Mallat's WT algorithm was applied to the image shown on Fig.1a. The texture maps were built by adding quadratically the detail images and normalizing by the low-resolution approximation resulting from the algorithm. Fig.1b is the texture mapy at scale $200 \mathrm{~m}$. As the scale increases, the discontinuities in the signal are enhanced. 
a)
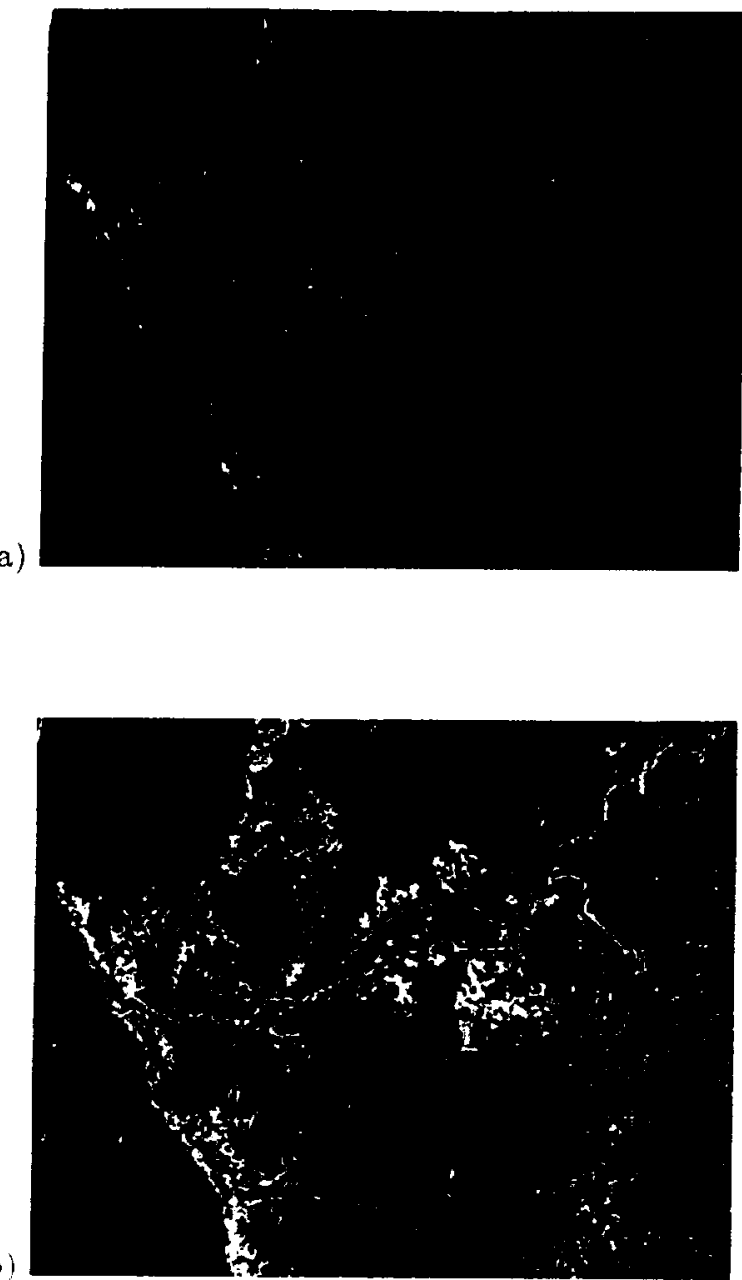

Fig. 1. a) SAR image of part of Gabon. Training sites are shown by arrows. b) Texture map of the region at scale $200 \mathrm{~m}$.

Two dates were input into the classifier as well as the texture maps for the low water season. Fig.2 shows the linear histogram stretch applied to the data to cover the range of byte values $(0-255)$. A set of 15 classes was chosen along with large training areas (Low land forest, medium topography forest. libghliud fortst, raphiat, inundated, water area, rivir. lity. saviunat, savilua teluporal change, swamp, regrowth. low mallgove, high mallgrove,open forest).

The intial tese was limited to a maximum of 7 levels. Pruniuf wat then applied to produce a sfupence of trees with varioll: complixity. Bufure: frowing the tree, $20 \%$ of the saluplos for eauth alasses wert: follserved. This fraction is

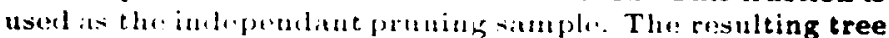
in shuwn in Fig. III. Tha: trew was rulued froul 128 terminal

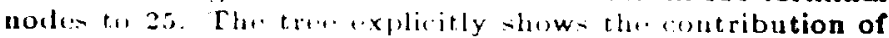

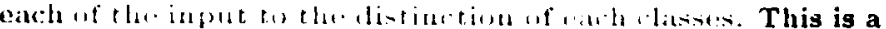

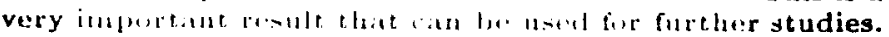

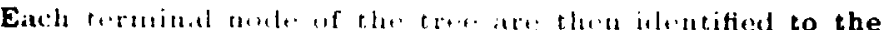

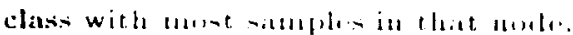

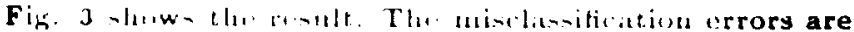

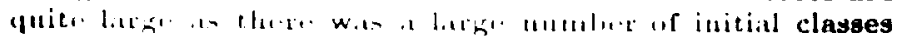

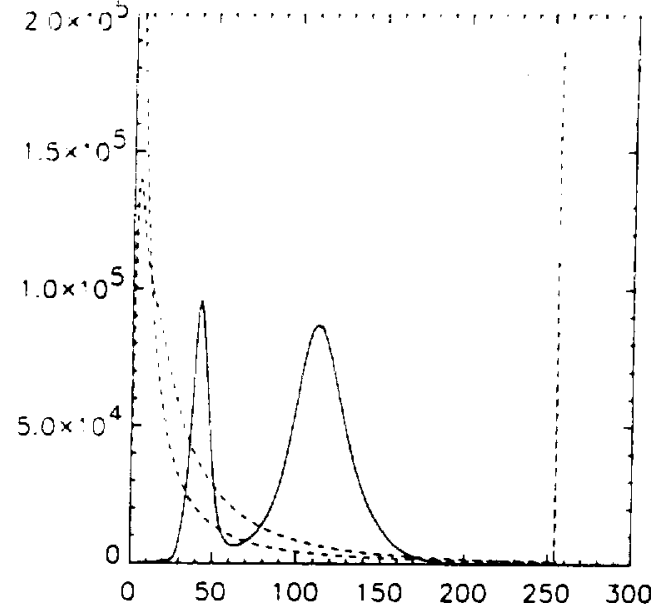

a)

Fig. 2. Histograms of the input images: Low water amplitude (Full line), textures (dashed lines).

(errors: Low land forest (10\%), medium topography forest $(23 \%)$, highland forest $(61 \%)$, raphia $(37 \%)$, inundated $(17 \%)$, water area $(29 \%$ mixed with savanna), river $(15 \%)$, city (24\%), savanna ( $50 \%$ with water), savanna temporal change ( $50 \%$ ), swamp ( $53 \%$ ), regrowth (classifled as forest), low mangrove (mostly classified as forest $90 \%$ ), high mangrove $(50 \%)$,open forest $(5 \%))$. Grouping of classes could significantly reduce errors.

\section{Conclusion}

The results explicitly demonstrate the contribution of each input to distinction between the classes. It also shows the limitations of using JERS-1 SAR configuration for monitoring of tropical forests. That is, it is limited to biomass extremes (ex: savanna-forest) and vegetation moisture (e.g. inundated areas). The decision tree classifier can be used with different inputs. Thus, studies with different texture measures will be performed. We also need to compare this classiffer to classical ones such as the ML method.

The result errors and artefact demonstrate the importance of choosing the training sites. This is readily seen when comparing misclassification Thus, these result must be considered when choosing the training areas. misclassification high land forest as edges (or open forest). This is due to very large texture.

\section{REFERENCES}

[1] S. Sastchi, J. Soares, and D. Alves, "Mapping deforestation and land use in amazon rainforest by using sir-c imagery," Remote Sensing of the Environment, vol. 59, no. 2, pp. 191-202, 1997.

[2] J.-P. Malingreau and G. Duchossois, "The TREES/ ERS-1 SAR'94 project," Earth observation quar. terly, ESt,http://esupub.esrin.esa.it/eoq, vol. 48, pp. 1-5. 1995.

[3] L. Breiman, J. H. Friedman, R. A. Olshen, and C. J. Stone. Clissification ind Regression Trees. Chapman and HallBerlin. New-York London., 1984

(1) S. Mallat, "A theory for multi-resolution signal decomposition: The wavelet representation," IEEE Transactions on pattern and muchine inleliyence, vol. 11, no. 7 . pp. 671-693, 1989. 


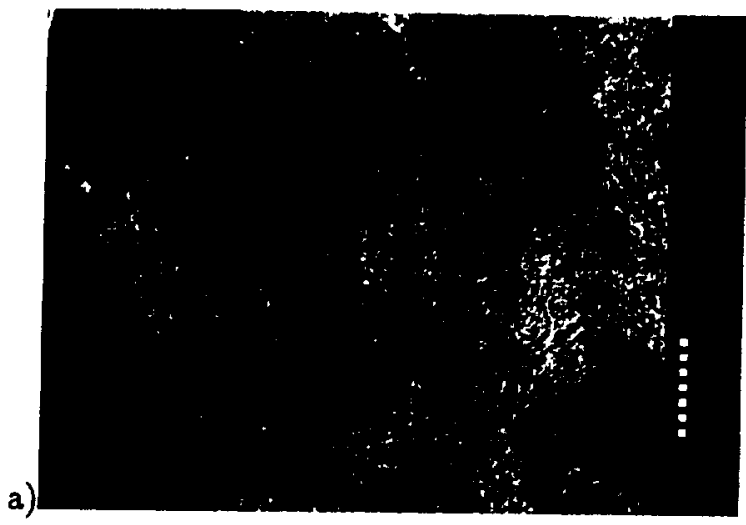

Fig. 3. Classified map of Gabon. The end nodes resulting from the algorithm have been grouped (in colors) to the class with largeat proportion. Water (class 0), low land forest (class), medium topography forest (class) High land forest (class), raphia (class), inundated vegetation (class), savanna (class), Temporal change savanna (class).

[5] M. Simard, F. DeGrandi, K. Thomson, and G. Bénié, "Analyse multi-échelle de la texture de la mosaique ERS-1 de la forèt tropicale africaine," CDROM du $9^{e}$ congrés de l'association québécoise de télédétection, Québec, 1996.

[6] M. Simard, "Extraction of information and speckle noise reduction in sar image using the wavelet transform," IGARSS'98 proceedings, 1998.

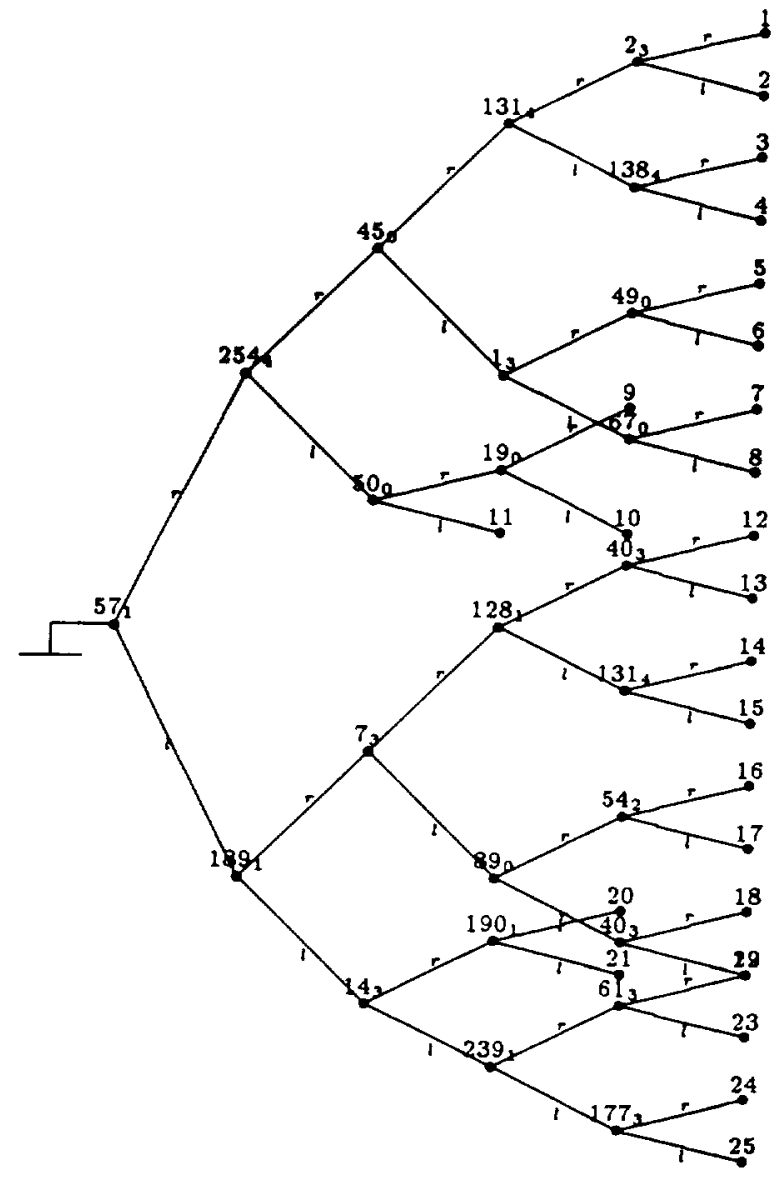

Fig. 4. Tree grown using training sample overall probabilities and pruning using one-class probabilities. At each node, a value correpondaing to the best split s threshold is given with the input parameter $i\left(s_{i}\right)$. The parameters are the amplitudes for low water (0), high water (1), texture $100 \mathrm{~m}(2), 200 \mathrm{~m} \mathrm{(3)}$ and 400 m (4). 